\title{
Determinants of Breast Self-Examination Performance Among Iranian Women: An Application of the Health Belief Model
}

\author{
Azita Noroozi - Tayyebh Jomand • Rahim Tahmasebi
}

Published online: 22 September 2010

(C) Springer 2010

\begin{abstract}
Breast cancer is one of the most common cancers among women. Screening behavior rates are low in the world. Therefore, the purpose of the current study was to investigate breast self-examination (BSE) rate and the relationships of Health Belief Model (HBM) constructs for predicting BSE. Path analysis was used to examine both one-way direct and indirect effects of HBM factors on BSE in this population $(N=382)$. Data were collected by a part of Champion's HBM Scale (CHBMS) and a selfadministered questionnaire. The results showed that 7.6\% of the participants reported performing BSE regularly. The final model provided a good fit to the data, with 13 variables explaining $62 \%$ of the variance in BSE. Perceived self-efficacy was intermediate construct between modifying factors and HBM constructs. Also, perceived self-efficacy and perceived benefits were the most highly related to BSE. The results suggest that HBM is a useful framework for identifying factors influencing the use of BSE in Iranian women.
\end{abstract}

This work was financially supported by Bushehr University of Medical Sciences, Bushehr, Iran

\footnotetext{
A. Noroozi $(\square)$

Department of Health, Bushehr University of Medical Sciences, Bushehr, Iran

e-mail: azitanoroozi@yahoo.com

T. Jomand

Department of Nursing, Bushehr University of Medical Sciences, Bushehr, Iran

R. Tahmasebi

Department of Biostatistics, Bushehr University of Medical

Sciences,

Bushehr, Iran
}

Keywords Breast cancer B Breast self-examination · Health Belief Model (HBM) · Iranian women

\section{Introduction}

The increased incidence of breast cancer has brought this disease to the consideration and concern of health professionals, as there were in the 2004 over one million new cases of breast cancer in the world. Furthermore, its rate in women living in the developing countries is rising [18].

In the Islamic Republic of Iran, breast cancer ranks as the first cancer among women, comprising $21.4 \%$ of all cancer malignancies among the Iranian females [21]. It affects the women at least one decade earlier than those in the developed countries [16]. One main reason for the continuous growth of breast cancer death rate among Iranian women is low screening rates and late detection of breast cancer [11, 16, 24].

Efforts at increasing the early breast cancer detection rate in women are important to improve women's health and to decrease the cost related to cancer death. Empirical evidence suggests that use of breast self-examination, clinical breast examination and mammography help to ensuring the early detection of breast cancer [17]. According to American Cancer Society recommendations, women should be familiar with how their breasts normally feel, and report any breast changes promptly to their health care providers [1], because $95 \%$ of breast cancers in advanced stages and $65 \%$ of breast cancers in primary stages are detected by the women [6]. Although the ACS no longer recommends breast self-examination (BSE; as there is reliable data that use of BSE does not increase survival rates), it should be noted that BSE is an important viable screening method for those living in rural areas or 
economically and culturally poor regions, where access to $\mathrm{CBE}$ and mammograms are difficult. In these cases, BSE may detect breast cancer early enough to enable treatment that prolongs women's lives and reduces suffering. Also for younger women, BSE training and practice is a gateway to health promotion behavior that provides women with knowledge and attitudes that set the stage for $\mathrm{CBE}$ and mammography screening later in life [3]. Also a meta-analysis study showed that women who performed BSE were more likely to seek medical advice and have biopsies [15]. The results of previous study indicated a gradual decrease in BSE performance, as the rates of BSE performance in Turkish women were $32.5 \%$ in $1985,20-28 \%$ in 2003 , and $17 \%$ in 2006 [28]. Thus, regular BSE remains an essential aspect of secondary prevention in Iran. Furthermore, understanding Iranian women's health beliefs related to breast cancer screening behaviors will help nurses and other healthcare professionals to choose more effective health education programs and thus potentially increase screening practices.

Cognitive factors play an important role in understanding health beliefs especially in BSE [9]. The Health Belief Model (HBM) is a cognitive model [8]. Champion modified it to examine the beliefs related to BSE practices by women [10]. This model suggests that changes in preventive health behaviors are originally based on four factors: (a) susceptibility: perceived personal vulnerability to or subjective risk of a health condition, (b) seriousness: perceived personal harm of the condition, (c) benefits: perceived positive attributes of an action, and (d) barriers: perceived negative aspects of an action. Two other concepts, health motivation and self-efficacy, were later added to the original HBM. Health motivation refers to the beliefs and behaviors related to the state of general concern about health. Perceived self-efficacy (confidence) is defined as this belief that one can successfully execute a behavior that will ultimately lead to a desirable outcome [7]. However, when the focus is the behaviors requiring long term changes such as BSE that should be performed monthly, self-efficacy is added to the model [13].

Furthermore, HBM has modifying factors. The ultimate decision to engage in the behavior is influenced by modifying factors, which may include demographic variables (such as age, educational level) and structural variables (such as knowledge about the condition or disease) [4].

In the previous studies, age, level of education, a referral from a physician, health insurance coverage, family history of breast cancer, and knowledge of breast cancer have also been associated with BSE $[20,22,26]$.

To provide a more comprehensive test of the HBM, in the current study we investigated the predictive value of HBM constructs and modifying factors, notably family history and demographic factors (i.e., age, educational level, marital status, etc.) in BSE performance.
In the developing countries as Iran, little is known about the factors associated with BSE performance and screening rates among the Iranian women.

Although HBM clearly identifies the constructs that lead to outcome behaviors, the relationships among the constructs are less clear [13]. Therefore, the purpose of the current study was to investigate BSE rate and the relationships of HBM constructs for predicting BSE and also to assess the predictive power of this model. The specific objectives of the study are:

1- Examining whether the modifying factors predict BSE behavior,

2- Examining whether HBM constructs predict breast self-examination behavior directly.

\section{Method}

Sample

The total of 403 women at work and in public places of Bushehr city (Iran) were recruited through convenience sampling procedure. The inclusion criteria were older than 18 years, not pregnant or breastfeeding, lacking mental and/or physical disabilities, and having the ability to read and write. The aim of the study was verbally explained to the potential participants that had inclusion criteria. Then they were asked if they agreed to participate in this study. The participants were told that all information would be kept secret and anonymous. They were also requested to choose the answer that best described their beliefs and opinions. The participants completed questionnaires right away on the site. From the total of 403 women, 21 participants submitted imperfect data questionnaire, so they were excluded from the study. The final sample included in the path analysis was 382 , yielding a $94.8 \%$ response rate.

\section{Instruments and Measures}

A self-administered questionnaire and a part of the Champion's Health Belief Model Scale (CHBMS) were used as the data collection instruments [7]. The questionnaire was used to obtain the information about the participants' socio-demographic characteristics and other factors such as having heard/read about breast cancer, sources of breast cancer information, and having a family history of breast cancer (this variable was scored as $1=$ having family history or $2=$ no family history). The sociodemographic variables (modifying factors) included age, menarche age, current marital status, years of education, employment status, contraception method used, gravidity, menopausal status, and health insurance coverage. The 
questionnaire was developed by the authors based on an extensive review of the literature.

Cancer knowledge questions included 24 questions, of which, 15 questions were about risk factors and nine others about the signs and symptoms of breast cancer. The answers for risk factor questions were 'increase', 'decrease', 'no effect' and 'I do not know', these questions were re-coded into dichotomous variables by coding false and $I$ do not known $=0$ and true $=1$. The answers for sign and symptom questions were yes $=1$ and no $=0$.

The HBM constructs including perceived susceptibility, perceived seriousness, perceived benefits, perceived barriers, self-efficacy, and health motivation were measured using the CHBMS. Six subscales of CHBMS with 42 items were used: susceptibility to breast cancer (five items), seriousness of breast cancer (seven items), benefits of breast exams (6 items), barriers to self-examination (six items), self-efficacy in one's ability to examine the breasts correctly (11 items), and health motivation that being concerned about health in general (seven items). All the items had a 5-point Likert scale ranging from strongly disagree (1-point) to strongly agree ( 5 points). In CHBMS, all of the scales were positively related to the screening behavior, except for the barriers which were negatively associated.

The dependent variable, practices related to BSE, was assessed by self-report on the frequency of breast exam. The BSE score was coded in non-practice $=1$, when I'm anguished $=2$, irregular practice $=3$, and regular practice (monthly) $=4$.

All the instruments were first translated by the principal researcher and a bilingual person then validated by the back translation technique. The translated instruments were reviewed by a group of Iranian health education experts. In this study, no items were changed. Prior to data collection the questionnaires relation to the CHBMS and the individual knowledge were tested for reliability in a sample of 50 Iranian women. All of the questionnaires were reliable and the ranges of Cronbach's alpha coefficients were 0.75 (health motivation) to 0.90 (susceptibility).

The mean scores (with standard deviations), range, and internal consistency of the constructs and knowledge are listed in Table 1.

\section{Data Analysis}

The obtained data were analyzed by path analysis using LISREL, version 8.80. Path analysis is used as a method for studying the direct and indirect effects of variables and for estimating the values of the coefficients in the underpinning linear model [27]. Mardia's coefficient for multivariate skewness was 26.57 and for kurtosis, it was estimated to be 9.17. These values were significant, therefore, some of the variables were non-normal. Therefore, we used robust maximum likelihood estimation procedure. A correlation matrix and an asymptotic covariance matrix were applied to model estimation.

Chi-square $\left(\chi^{2}\right)$, the adjusted goodness-of-fit index (AGFI) and the root-mean-square error of approximation (RMSEA) were used as model fit criteria. The model was considered fit if AGFI value was greater than 0.8 and if RMSEA value was less than 0.08 .

The comparative fit index of Bentler-Bonett nonnormed fit index (NNFI) was selected. Values of 0.90 or greater for NNFI are recommended as acceptable values for this measure.

$\mathrm{T}$ value was used for elimination of the parameters in the path analysis, and Modification index was used for inclusion of additional parameters.

\section{Results}

The total of 382 participants were included in the study with the age range of 20-66 years old (median=32, M= $34.15, \mathrm{SD}=10.6)$. Most of the participants were married (73.6\%). $19.2 \%$ of the respondents $(n=73)$ were educated in primary/secondary level, $41 \%(n=157)$ were graduated from high school and 39.8\% $(n=152)$ had obtained college degree. Many the participants were housekeeper and jobholder (46.9\% and $36.6 \%$, respectively). Their gravidity ranged from 0 to $12(\mathrm{M}=2.08, \mathrm{SD}=2.3)$. The majority of the participants $(51.6 \%)$ did not use any contraception methods, $8.6 \%(n=33)$ had withdrawal contraception and $39.8 \%(n=152)$ used modern contraception methods such as oral contraception pills, IUD and injection methods. Only $10.8 \%(n=40)$ of the participants were in menopause stage. Regarding the family history, $87.2 \%$ of the women $(n=333)$ had no known familial history of breast cancer. $58.1 \%$ of the respondents $(n=222)$ stated that they did not perform BSE, and only $7.6 \%$ of them $(n=29)$ reported that they were practicing BSE regularly (monthly). $34.3 \%$ of the participants did not receive any information about breast cancer. Among the women who received information about breast cancer, radio and television were identified as the main sources of information on BSE by $37.8 \%$ of the participants $(n=95)$. Health professionals (doctors and nurses; $19.1 \%)$ and printed materials $(25.5 \%)$ were also identified as the other sources of information on BSE.

Average responses for the six belief scales are summarized in Table 1. The results (according to mean values obtained) demonstrated that the participants in this study believed that they had low levels of susceptibility to breast cancer, barriers to performance of BSE, and self-efficacy of BSE. Also, the women perceived medium levels of seriousness, benefits of BSE, and health motivation. They also had low level of knowledge related to breast cancer. 
Table 1 Means and standard deviations of the constructs $(N=382)$ and internal consistency $(N=50)$

\begin{tabular}{llllllll}
\hline Variables & Knowledge & Susceptibility & Seriousness & Benefits & Barrier & Self-efficacy & Health motivation \\
\hline Mean & 6.45 & 5.32 & 15.05 & 17.89 & 6.75 & 23.60 & 21.71 \\
Standard deviation & 5.11 & 4.37 & 6.52 & 4.47 & 4.79 & 8.58 & 4.21 \\
Range & $0-24$ & $0-25$ & $0-35$ & $0-30$ & $0-30$ & $0-66$ & $0-35$ \\
Cronbach's alpha & 0.87 & 0.90 & 0.85 & 0.85 & 0.82 & 0.89 & 0.75 \\
\hline
\end{tabular}

Correlation coefficients including polyserial or Pearson's coefficients are given in Table 2. The variables including seriousness of breast cancer, barriers to self-examination, and self-efficacy to examine the breast were significantly related to BSE among Iranian women. A positive association was found between BSE and self-efficacy to examine the breast and a negative association was found between BSE and seriousness of breast cancer and barriers to BSE.

\section{Structural Model}

The original hypothesized model did not result in a good fit to the data $\left(\chi^{2}=413.37, d f=15, P<0.001\right.$, RMSEA $=0.262$, $\mathrm{NFI}=0.88, \mathrm{GFI}=0.88, \mathrm{AGFI}=-0.37$ ). Examination of the coefficients of the paths in the hypothesized model revealed that some coefficients were significant and some were not. After considering the results of the original model and the related theoretical issues, the model was modified by adding five paths one-by-one between the constructs and dropping several paths with non-significant coefficients one-by-one between the modifying factors (i.e., knowledge about breast cancer, employment status, contraception method, gravidity, and health insurance coverage) and model constructs. The fit indices indicated improvement of the modified model over the original model $\left(\chi^{2}=171.97, \mathrm{df}=62, P<0.001\right.$, RMSEA $=$ $0.068, \mathrm{NFI}=0.94, \mathrm{GFI}=0.96$, AGFI $=0.87)$. The coefficients between the variables were improved and all the paths in the modified model were significant. Although the Chi-square statistics indicated that the null hypothesis for this study was rejected, Chi-square statistics are known to be sensitive to a large sample size [27]. Other fit indices such as GFI and AGFI that had not been affected by sample size indicated a good fit of the model (values $>0.9$ ).

The final path model with standardized coefficients is depicted in Fig. 1.

The results indicated that there was a significant positive direct effect of age on BSE. Among the modifying factors, age had the most affect in BSE performance with the factor loading of 0.826 . Perceived susceptibility (of individual perception component) only had a significant negative direct effect on BSE. Furthermore, there were positive direct and indirect effects of perceived benefits, seriousness, and selfefficacy on BSE. Also, perceived barrier had negative direct and indirect effects on BSE. The results indicated that all of constructs except health motivation directly influenced BSE. The self-efficacy had direct effect in BSE performance. This construct also affected perceived barriers, benefits, seriousness, and susceptibility. In this study, the most effective factor in BSE performance was self-efficacy with the highest factor loading (0.264). Direct and indirect influences of the modifying factors and HBM constructs on BSE are shown in Tables 3 and 4, respectively.

This model accounted for $62 \%$ of the variance in participation in BSE among Iranian women.

\section{Discussion}

In this study, only $7.7 \%$ of the participants reported that they practiced BSE on a regular (monthly) basis. A study

Table 2 Correlation coefficients among the constructs and knowledge

\begin{tabular}{|c|c|c|c|c|c|c|c|c|}
\hline & Knowledge & Susceptibility & Seriousness & Benefits & Barrier & Self-efficacy & Motivation & BSE \\
\hline Knowledge & 1 & & & & & & & \\
\hline Susceptibility & 0.44 & 1 & & & & & & \\
\hline Seriousness & -0.003 & $0.3 * *$ & 1 & & & & & \\
\hline Benefits & $0.25 * *$ & 0.045 & $0.25 * *$ & 1 & & & & \\
\hline Barrier & $-0.26 * *$ & 0.09 & $0.27 * *$ & $-0.19 * *$ & 1 & & & \\
\hline Self-efficacy & $0.28 * *$ & 0.01 & -0.08 & $0.32 * *$ & $-0.24 * *$ & 1 & & \\
\hline Motivation & 0.1 & 0.04 & -0.09 & $0.24 * *$ & $-0.23 * *$ & $0.27 * *$ & 1 & \\
\hline BSE & 0.12 & 0.04 & $-0.14^{*}$ & 0.11 & $-0.14 *$ & $0.22 * *$ & 0.11 & 1 \\
\hline
\end{tabular}

${ }^{*} P<0.05$ (two-tailed); ${ }^{* *} P<0.01$ (two-tailed) 


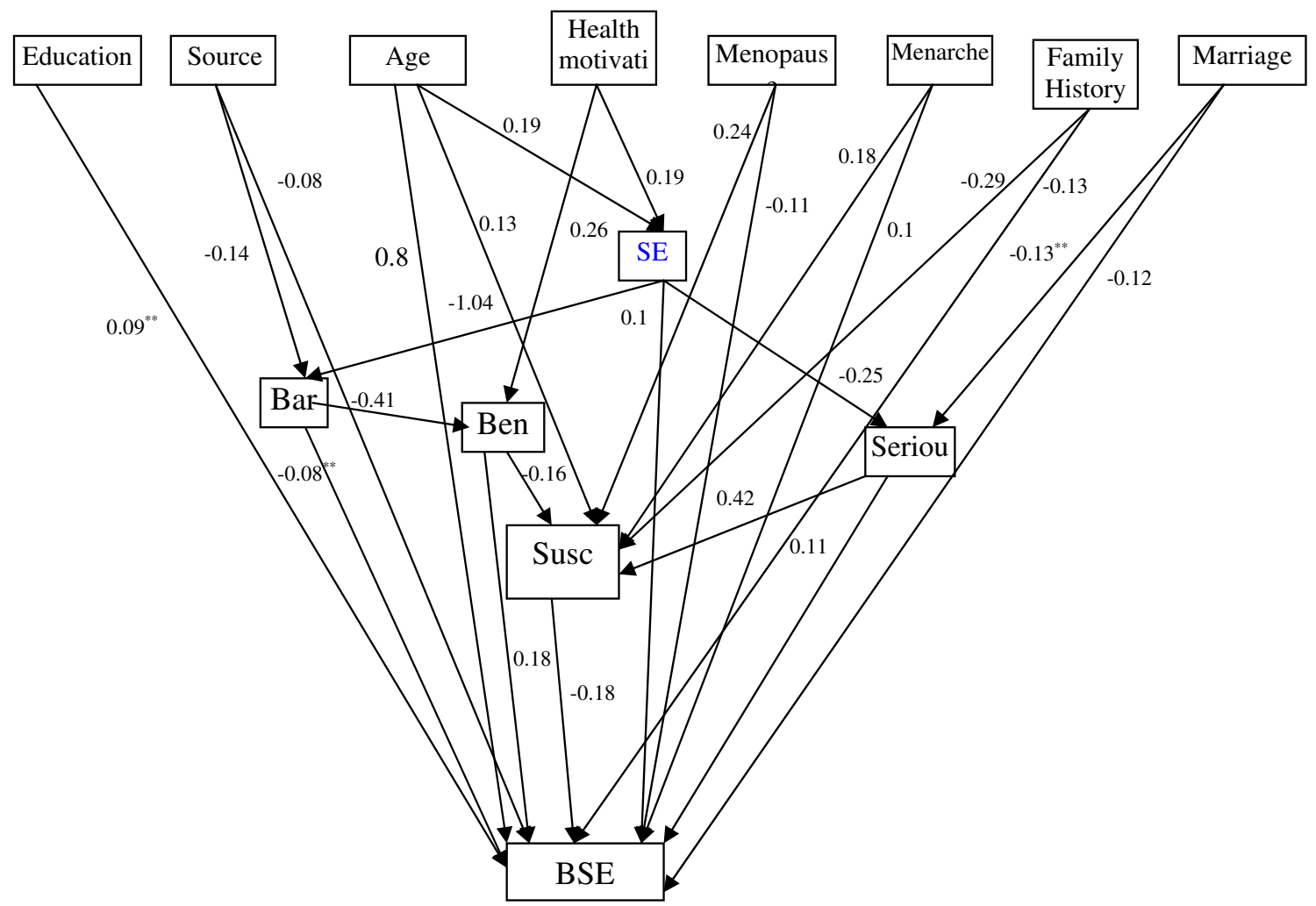

Fig. 1 The final version of HBM for breast self-examination. Standardized coefficients with double asterisks have $P<0.01$, standardized coefficients without asterisk have $P<0.001, R^{2}=0.62$. Note: $B S E$ breast self-examination, Susc perceived susceptibility to breast

on the BE practices among the Jewish and Arab women indicated that significantly more Jewish women (26.8\%) than Arab women $(22.3 \%)$ had ever performed a regular BSE [10]. A study on community samples of diverse women groups in USA, the rate of performing monthly BSE ranged from $29 \%$ to $63 \%$ [28]. In a sample of Turkish Muslim women, only $4.3 \%$ of the participants reported that they practised BSE regularly [3]. Thus Iranian women similar to Turkish Muslim women performed less BSE regularly than other women in the world. Therefore, delineating the cognitive correlates of BSE is essential to the development of effective interventions.

The results of this research support the direct or indirect effects of HBM constructs on BSE performance among Iranian women. Some demographic variables including: age, information sources, having a family history of breast cancer, current marital status, years of education, menarche, and menopausal status predicted BSE performance.

The results of this study are consistent with the findings of other studies. Many studies have found perceived selfefficacy as the most powerful and consistent predictor of BSE $[3,19,28,29]$. Self-efficacy was the core construct in our study. This construct had direct effect in BSE performance. It also indirectly influenced BSE performance cancer, Seriou perceived seriousness of breast cancer, Ben benefits of breast exams, Bar barriers to self-examination, $S E$ self-efficacy to breast self-examination, Source information sources

through impressing perceived barrier, benefit, seriousness, and susceptibility.

Perceived benefits of BSE was the second predictor on BSE performance in this study. The women who perceived more benefits of breast self-exam reported frequent selfexams. In several studies, similar to our study, perceived benefits have been found to be significantly related to BSE $[26,29]$.

Seriousness of breast cancer was the weakest predictor for performing BSE. This result is in accordance to findings of other studies. In some studies found that it was not significantly associated with performing BSE $[3,28]$. One explanation for this finding may be that breast cancer is regarded as a serious condition by most women [12], as the women in this study perceived more seriousness than other constructs.

In contrast to previous studies [28, 29], perceived susceptibility was a negative significant predictor for BSE performance in our study, as women who perceived greater susceptibility performed fewer BSE. It is possible that susceptible women view the quality of breast selfexamination as much more relevant to tumor detection than its mere frequency [29]. Also in the present study, perceived susceptibility was influenced by perceived 
Table 3 Direct and indirect influences of modifying factors on BSE

\begin{tabular}{|c|c|c|c|c|}
\hline \multirow{2}{*}{$\begin{array}{l}\text { Predictor } \\
\text { variables }\end{array}$} & \multirow[t]{2}{*}{ Through } & \multicolumn{3}{|c|}{ Causal effect } \\
\hline & & Direct & Indirect & Total \\
\hline \multirow[t]{9}{*}{ Age } & - & 0.8 & - & \\
\hline & Susc & - & -0.023 & \\
\hline & SE, Seriou & - & -0.005 & \\
\hline & SE, Seriou, Susc & - & 0.003 & \\
\hline & $\mathrm{SE}$ & - & 0.019 & \\
\hline & SE, Bar & - & 0.016 & \\
\hline & SE, Bar, Ben & - & 0.014 & \\
\hline & SE, Bar, Ben, Susc & - & 0.002 & \\
\hline & Total & 0.8 & 0.026 & 0.826 \\
\hline Education & - & 0.09 & - & 0.09 \\
\hline \multirow{5}{*}{$\begin{array}{l}\text { Information } \\
\text { source }\end{array}$} & - & -0.08 & & \\
\hline & Bar & - & 0.011 & \\
\hline & Bar, Ben & - & 0.01 & \\
\hline & Bar, Ben, Susc & - & 0.001 & \\
\hline & Total & -0.08 & 0.022 & -0.058 \\
\hline \multirow[t]{3}{*}{ Menarche } & - & 0.1 & & \\
\hline & Susc & - & -0.032 & \\
\hline & Total & 0.1 & -0.032 & 0.068 \\
\hline \multirow[t]{3}{*}{ Menopause } & - & -0.11 & - & \\
\hline & Susc & - & -0.043 & \\
\hline & Total & -0.11 & -0.043 & -0.153 \\
\hline \multirow[t]{3}{*}{ Family history } & - & -0.13 & - & \\
\hline & Susc & - & 0.052 & \\
\hline & Total & -0.13 & 0.052 & -0.078 \\
\hline \multirow[t]{4}{*}{ Marriage } & - & -0.12 & - & \\
\hline & Seriou & - & -0.014 & \\
\hline & Serious, Susc & - & -0.01 & \\
\hline & Total & -0.12 & -0.024 & -0.144 \\
\hline
\end{tabular}

Susc perceived susceptibility to breast cancer, Seriou perceived seriousness of breast cancer, Ben benefits of breast exams, Bar barriers to self-examination, $S E$ self-efficacy to self breast examination

seriousness of breast cancer, as the women who looked upon breast cancer as a more severe disease had perceived more susceptibility. Accordingly, since Iranian women look upon breast cancer as a terrible and catastrophic illness, they preferred to keep it uncovered, so susceptible women performed fewer BSE. Therefore, attitude change about breast cancer in these women is necessary.

Some studies have found perceived barriers as an important predictor of frequency of BSE [10, 29]. In this study, perceived barriers had direct and indirect effects on BSE performance. Women who perceived greater barriers to BSE were somewhat less likely to perform BSE and perceived fewer benefits for BSE. In this study, women who received information through television and radio rather than from medical staff perceived more barriers to BSE. Our data also demonstrated that television and radio were the main sources of information about breast cancer. This result is in agreement with the findings of other studies [25, 26]. Therefore, medical staff should establish a sincere and oneto-one communication with their clients about breast cancer screening for promoting BSE, because effective health communication has been found to be a very important motivating factor for screening behavior [5, 21].

Health motivation did not predict directly BSE performance, but it influenced self-efficacy and perceived benefits of BSE. Therefore, consistent to the theory of HBM and the findings of other studies [10], health motivation was found to be significantly associated with performing BSE in this study.

Table 4 Direct and indirect influences of HBM constructs on BSE

\begin{tabular}{|c|c|c|c|c|}
\hline \multirow{2}{*}{$\begin{array}{l}\text { Predictor } \\
\text { variables }\end{array}$} & \multirow[t]{2}{*}{ Through } & \multicolumn{3}{|c|}{ Causal effect } \\
\hline & & Direct & Indirect & Total \\
\hline \multirow{9}{*}{$\begin{array}{l}\text { Health } \\
\text { motivation }\end{array}$} & Ben & - & 0.047 & \\
\hline & Ben, Susc & - & 0.007 & \\
\hline & $\mathrm{SE}$ & - & 0.019 & \\
\hline & SE, Bar & - & 0.016 & \\
\hline & SE, Bar, Ben & - & 0.014 & \\
\hline & $\begin{array}{l}\text { SE, Bar, Ben, } \\
\text { Susc }\end{array}$ & - & 0.002 & \\
\hline & SE, Seriou & - & -0.005 & \\
\hline & SE, Seriou, Susc & - & 0.003 & \\
\hline & Total & - & 0.103 & 0.103 \\
\hline \multirow[t]{7}{*}{ Self-efficacy } & - & 0.1 & - & \\
\hline & Bar & - & 0.083 & \\
\hline & Bar, Ben & - & 0.077 & \\
\hline & Bar, Ben, susc & - & 0.012 & \\
\hline & Seriou & - & -0.027 & \\
\hline & Seriou, Susc & - & 0.019 & \\
\hline & Total & 0.1 & 0.164 & 0.264 \\
\hline \multirow[t]{3}{*}{ Seriousness } & - & 0.11 & - & \\
\hline & Susc & - & -0.076 & \\
\hline & Total & 0.11 & -0.076 & 0.034 \\
\hline Susceptibility & - & -0.18 & - & -0.18 \\
\hline \multirow[t]{3}{*}{ Benefits } & - & 0.18 & - & \\
\hline & Susc & - & 0.029 & \\
\hline & Total & 0.18 & 0.029 & 0.209 \\
\hline \multirow[t]{4}{*}{ Barriers } & - & -0.08 & - & \\
\hline & Ben & - & -0.074 & \\
\hline & Ben, Susc & - & -0.012 & \\
\hline & Total & -0.08 & -0.086 & -0.166 \\
\hline
\end{tabular}

Susc perceived susceptibility to breast cancer, Seriou perceived seriousness of breast cancer, Ben benefits of breast exams, Bar barriers to self-examination, $S E$ self-efficacy to self breast examination 
Similar to the findings of other studies [3, 28, 29], our findings showed among the modifying factors, age was the most important predictor for BSE practice, as older and also menopause women performed more BSE. These women perceived themselves less susceptible to breast cancer. Therefore, they were inclined to perform more BSE.

Consistent to the findings of a study [3], women's marital status was found to be predictor of performing BSE in Iranian women, as married women performed more BSE, but this result is in contradiction with the finding of some other studies [29]. Thus it seems still more investigations are required in this area.

Contrary to the findings of some studies $[9,29]$, but in accordance with some others $[2,10,15]$, having a firstdegree relative affected by breast cancer was not associated with higher practice of BSE, so that these women with family history of breast cancer even had fewer BSE. On the other hand, since they perceived themselves more susceptible to breast cancer, therefore, they preferred to keep it secret and performed fewer BSE.

Similar to other studies $[3,10]$, level of education was a predictor for BSE performance as well in this study.

In conclusion, increasing of self-efficacy using different techniques (i.e., verbal persuasion, emotional arousal, modeling, and performance accomplishments), emphasizing on the various benefits of BSE in the early detection of breast cancer, minimizing barriers to BSE, and modifying attitude about breast cancer by medical staff especially for younger women with the family history of breast cancer are necessary.

As for limitations of this study, conclusions from selfreport data also demand caution. Inaccurate recall might adversely affect self-reports of BE performance, which sometimes tend to be overestimated [23], nonetheless, some studies suggest that self-reports provide a fairly accurate indication of screening behaviors [14]. Since, this study was based on a convenience sample, so that its findings of this study may not be generalized to all Iranian women. Overall, community-based programs should be expanded to different Iranian women groups to assess the actual rate of screening behaviors and effective factors on them.

\section{Modifying factors}

Demographic variables

1. Age

2. Education level

(1) Primary school

(2) Secondary school

(3) High school

(4) University graduate
3. Women's job
(1) Housewife
(2) Worker
(3) Civil servant
(4) Private sector
(5) Student

4. Marital status
(1) Married
(2) Single
(3) Widow
(4) Separated

5. Perceived family income level
(1) Sufficient

(2) Insufficient

6. Health insurance

(1) Absent (2) Poverty card (3) State health insurance

(4) Private health insurance

7. Menarch age

8. History of menopause

(1) Yes (2) No (if the answer is Yes, age of menopause)

9. Information about breast cancer? (1) Yes (2) No (If 'Yes', source of information)

(1) No information (2) Health professionals (3) Books/brochures/magazins (4) Friends-neighborhood (5) TV-radio (6) Other

10. Family history of breast cancer (you, your mother, sister, aunt, grandmother)/your friends

(1) No (2) Yes, of my family (3) Yes, me (4) Yes, my friends

Questions for Married

11. Gravity

12. Number of live birth

13. Number of stillbirth

14. Number of abortus

15. Number of living child

16. Age at first delivery

17. Duration of breastfeeding (month)

18. Current family planning method

(1) Absent (2) Traditional method (coitus interruptus etc.) (3) Modern method

Structural variables (Questions About Knowledge Level of Breast Cancer):

19. What is the effect of aging on breast cancer probabililty?

(1) Increase (2) Decrease (3) No effect (9) Do not know 
20. What is the effect of nulliparity on breast cancer probability?
(1) Increase
(2) Decrease
(3) No effect (9) Do not know

28. What is the effect on breast cancer probability if first delivery age is above 30 ?

(1) Increase (2) Decrease (3) No effect (9) Do not know

29. What is the effect on breast cancer probability if menopaue age is above 50 ?

(1) Increase (2) Decrease (3) No effect (9) Do not know

30. What is the effect on breast cancer probability if menarch age is under 11 ?

(1) Increase (2) Decrease (3) No effect (9) Do not know

31. What is the probability of counter-lateral cancer formation in breast cancer patients?

(1) Increases (2) Decreases (3) No effect (9) Do not know

32. What is the effect on breast cancer probability if family history is present?

(1) Increase (2) Decrease (3) No effect (9) Do not know

33. What is the effect of obesity on breast cancer probability?

(1) Increase (2) Decrease (3) No effect (9) Do not know

34. What is the effect of oral contraceptives on breast cancer probability?

(1) Increase (2) Decrease (3) No effect (9) Do not know

35. What is the effect of breastfeeding on breast cancer probability?

(1) Increase (2) Decrease (3) No effect (9) Do not know

36. What is the effect of using alcohol on breast cancer probability?

(1) Increase (2) Decrease (3) No effect (9) Do not know

37. What is the effect of smoking on breast cancer probability?

(1) Increase (2) Decrease (3) No effect (9) Do not know

38. What is the effect of radiation exposure on breast cancer probability?

(1) Increase (2) Decrease (3) No effect (9) Do not know

39. What is the effect of having beningn breast disease on breast cancer probability?

(1) Increase (2) Decrease (3) No effect (9) Do not know
40. What is the effect of hormone replacement therapy on breast cancer probability?

(1) Increase (2) Decrease (3) No effect (9) Do not know

You have knowledge about of which of the following symptoms:

41. Bloody discharge from nipple. (1) Yes (0) No

42. Asymmetric sagging in breast. (1) Yes 0) No

43. Breast mass. (1) Yes (0) No

44. Enlargement of neighbouring lymph nodes. (1) Yes (0) No

45. Breast skin retraction (1) Yes 0) No

46. Abnormal arm swelling 1)yes 0 )no

47. Nipple retraction (1) Yes (0) No

48. Discoloration of breast. (1) Yes (0) No

49. Abnormal enlargement of breast. (1) Yes 0) No

50. Do you know breast self-examination? (1) Yes (2) No (If yes, frequency of application?)
a. Yes I know, but never applied
b. I apply whenever I am anguished
c. I perform irregularly
d. I perform regularly (monthly)

Champion's Revised Health Belief Model Scales

Scale Items

Susceptibility

1. It is extremely likely I will get breast cancer in the future.

2. I feel I will get breast cancer in the future.

3. There is a good possibility I will get breast cancer in the next 10 years.

4. My chances of getting breast cancer are great.

5. I am more likely than the average women to get breast cancer.

Seriousness

1. The thought of breast cancer scares me.

2. When I think about breast cancer, my heart beats faster.

3. I am afraid to think about breast cancer.

4. Problems I would experience with breast cancer would last a long time.

5. Breast cancer would threaten a relationship with my boyfriend, husband or partner.

6. If I had breast cancer my whole life would change.

7. If I developed breast cancer, I would not live longer than 5 years.

Benefits

1. When I do breast self-examination I feel good about myself.

2. When I complete monthly breast self-examination I do not worry as much about breast cancer. 
3. Completing breast self-examination each month will allow me to find lumps early.

4. If I complete breast self-examination monthly during the next year, I will decrease my chance of dying from breast cancer.

5. If I complete breast self-examination monthly, I will decrease my chances of requiring radical or disfiguring surgery if breast cancer occurs.

6. If I complete monthly breast self-examination, it will help me to find a lump which might be cancer before it is detected by a doctor or nurse.

\section{Barriers}

1. I feel funny doing breast self-examination.

2. Doing breast self-examination during the next year will make me worry about breast cancer.

3. Breast self-examination will be embarrassing to me.

4. Doing breast self-examination will take too much time.

5. Doing breast self-examination will be unpleasant.

6. I do not have enough privacy to do breast selfexamination.

\section{Sel-Efficacy}

1. I know how to perform breast self-examination.

2. I am confident I can perform breast self-examination correctly.

3. If I were to develop breast cancer, I would be able to find a lump by performing breast self-examination.

4. I am able to find a breast lump if I practice breast selfexamination.

5. I am able to find a breast lump which is the size of a quarter.

6. I am able to find a breast lump which is the size of a dime.

7. I am able to find a breast lump which is the size of a pea.

8. I am sure of the steps to follow for doing breast selfexamination.

9. I am able to identify normal and abnormal breast tissue when I do breast self-examination.

10. When looking in the mirror, I can recognize abnormal changes in my breast.

11. I can use the correct part of my fingers when I examine my breasts.

Health Motivation

1. I want to discover health problems early.

2. Maintaining good health is extremely important to me.

3. I search new information to improve my health.

4. I feel it is important to carry out activities which will improve my health.

5. I eat well-balanced meals.

6. I exercise at least three times a week.

7. I have regular health check-ups even if I am not sick.

\section{References}

1. American Cancer Society (2000). How many women get breast cancer? Available at: http://www.cancer.org/docroot/CRI/content/ CRI_2_2_1X_How_many_people_get_breast_cancer_5.asp? siteareas. Accessed 24 Nov 06)

2. Andersen MR, Smith R, Meischke H, Bowen D, Urban N (2003) Breast cancer worry and mammography use by women with and without a family history in a population-based sample. Cancer Epidemiol Biomarkers Prev 12(4):314-320

3. Avci IA (2008) Factors associated with breast self-examination practices and beliefs in female workers at a Muslim community. Eur J Oncol Nurs 12(2):127-133

4. Battler JT (2001) Principles of health education and health promotion, 3rd edn. Thomson Learning Inc, CA, USA

5. Bener A, Honein G, Carter AO, Da'ar Z, Miller C, Dunn EV (2002) The determinants of breast cancer screening behavior: a focus group study of women in the United Arab Emirates. Oncol Nurs Forum 29(9):E91-E98

6. Champion V (1992) The role of breast self-examination in breast cancer screening. Cancer 1(7):1985-1991

7. Champion VL (1993) Instrument refinement for breast cancer screening behaviors. Nurs Res 42(3):139-143

8. Champion VL (1999) Revised susceptibility, benefits, and barriers scale for mammography screening. Res Nurs Health 22(4):341-348

9. Cohen M (2002) First-degree relatives of breast-cancer patients: cognitive perceptions, coping, and adherence to breast selfexamination. Behav Med 28(1):15-22

10. Cohen M, Azaiza F (2005) Early breast cancer detection practices, health beliefs, and cancer worries in Jewish and Arab women. Prev Med 41(5-6):852-858

11. Ebrahimi M, Vahdaninia M, Montazeri A (2002) Risk factors for breast cancer in Iran: a case-control study. Breast Cancer Res 4(5):R10

12. Fung SY (1998) Factors associated with breast self-examination behaviour among Chinese women in Hong Kong. Patient Educ Couns 33(3):233-243

13. Glanz K, Rimer BK, Lewis FM (2002) Health behavior and health education: theory, research, and practice, 3rd edn. Wiley, San Francisco

14. Gordon NP, Hiatt RA, Lampert DI (1993) Concordance of selfreported data and medical record audit for six cancer screening procedures. J Natl Cancer Inst 85(7):566-570

15. Hackshaw AK, Paul EA (2003) Breast self-examination and death from breast cancer: a meta-analysis. Br J Cancer 88(7):1047-1053

16. Harirchi I, Karbakhsh M, Kashefi A, Momtahen AJ (2004) Breast cancer in Iran: results of a multi-center study. Asian Pac J Cancer Prev 5(1):24-27

17. Harris JR, Morrow M, Osbome C (2002) Diseases of the breast, 2nd edn. Lippincott Williams and Wilkins, Philadelphia

18. International Agency for Research on Cancer (2006). CANCER mondial [online data-base] (http://www-dep.iarc.fr/. Accessed 7 Dec 2006

19. Jirojwong S, MacLennan R (2003) Health beliefs, perceived selfefficacy, and breast self-examination among Thai migrants in Brisbane. J Adv Nurs 41(3):241-249

20. Juon HS, Seo YJ, Kim MT (2002) Breast and cervical cancer screening among Korean American elderly women. Eur J Oncol Nurs 6(4):228-235

21. Lamyian M, Hydarnia A, Ahmadi F, Faghihzadeh S, Aguilar-Vafaie ME (2007) Barriers to and factors facilitating breast cancer screening among Iranian women: a qualitative study. East Mediterr Health J 13 (5):1160-1169

22. Legg JS, Fauber TL, Ozcan YA (2003) The influence of previous breast cancer upon mammography utilization. Womens Health Issues 13(2):62-67

23. McCaul KD, Branstetter AD, Schroeder DM, Glasgow RE (1996) What is the relationship between breast cancer risk and mam- 
mography screening? A meta-analytic review. Health Psychol 15 (6):423-429

24. Montazeri A, Ebrahimi M, Mehrdad N, Ansari M, Sajadian A (2003) Delayed presentation in breast cancer: a study in Iranian women. BMC Womens Health 3(1):4

25. Petro-Nustas W (2001) Young Jordanian women's health beliefs about mammography. J Community Health Nurs 18(3):177-194

26. Petro-Nustus W, Mikhail BI (2002) Factors associated with breast self-examination among jordanian women. Public Health Nurs 19 (4):263-271
27. Schumacker RE, Lomax RG (2004) A beginner's guide to structural equation modeling, 2nd edn. Lawrence Erlbaum Associates, Mahwah

28. Secginli S, Nahcivan NO (2006) Factors associated with breast cancer screening behaviours in a sample of Turkish women: a questionnaire survey. Int J Nurs Stud 43(2):161171

29. Umeh K, Dimitrakaki V (2003) Breast cancer detection in asymptomatic women: health beliefs implicated in secondary prevention. J Appl Biobehav Res 8(2):96-115 